\title{
Extended Cahill-Glauber formalism for finite-dimensional spaces: I. Fundamentals
}

\author{
M. Ruzzi and D. Galetti \\ Instituto de Física Teórica, Universidade Estadual Paulista, \\ Rua Pamplona 145, 01405-900, São Paulo, SP, Brazil \\ E-mail address: mruzzi@ift.unesp.brand galetti@ift.unesp.br \\ M. A. Marchiolli \\ Instituto de Física de São Carlos, \\ Universidade de São Paulo, \\ Caixa Postal 369, 13560-970, São Carlos, SP, Brazil \\ E-mail address: marcelo_march@bol.com.br
}

(Dated: September 6, 2018)

\begin{abstract}
The Cahill-Glauber approach for quantum mechanics on phase-space is extended to the finite dimensional case through the use of discrete coherent states. All properties and features of the continuous formalism are appropriately generalized. The continuum results are promptly recovered as a limiting case. The Jacobi Theta functions are shown to have a prominent role in the context.
\end{abstract}

\section{INTRODUCTION}

The search for discrete quantum phase-space quasiprobability distribution functions is a subject of continuous and growing interest in the literature [1, 2, 3, 4, 5, 6, 7, 8, 9, 10, 11, 26, 27]. The possibility of representing quantum systems characterized by a finite-dimensional state space by such discrete quasidistributions lays the ground for interesting developments and fruitful applications on quantum computation and quantum information theory 12, 13, 14, 15, 16, 17, 18, 19]. It is well known that, as a well established counterpart to the discrete case, a huge variety of quasiprobabilty distribution functions can be defined upon continuous phase-space [20]. In this sense, the Cahill-Glauber (CG) approach 21] to the subject has proved to be a powerful mapping technique that provides a general class of quasiprobability distribution functions, where the Wigner, Glauber-Sudarshan and Husimi functions appear as particular cases. Therefore, it might be considered as a wide-range phase-space approach to quantum mechanics regarding degrees of freedom with classical counterparts.

The aim of this paper is to present a discrete extension of the CG approach. Such extension is not obtained from that approach but, instead, properly constructed out of the finite dimensional context. Furthermore, this ab initio construction inherently embodies the discrete analogues of the desired properties of the CG formalism. In particular, discrete Wigner, Husimi and Glauber-Sudarshan quasiprobability distribution functions are obtained. Thus, besides the theoretical interest of its own, such extension has direct applications in quantum information processing, quantum tomography and quantum teleportation, which are explored in a following work 22 .

This work is organized as follows: In the next section we briefly outline the CG approach, setting the stage for section III, where our proposal for a discrete extension of the CG mapping kernel is presented. In section IV basic properties of the mapping technique are discussed, and the continuum limit is carried out on section V. Finally, section VI contains our summary and conclusions. Also, important calculations are detailed in the Appendix.

\section{THE CAHILL-GLAUBER MAPPING KERNEL}

For the sake of clarity, in what follows we will briefly review the central ideas which constitute the core of the CG approach, and that will be properly generalized in the following sections. Basically, the cornerstone of the formalism is the mapping kernel (hereafter $\hbar=1$ )

$$
\mathbf{T}^{(s)}(q, p)=\int_{-\infty}^{\infty} \frac{d p^{\prime} d q^{\prime}}{2 \pi} \exp \left[-i p^{\prime}(q-\mathbf{Q})\right] \exp \left[i q^{\prime}(p-\mathbf{P})\right] \exp \left(-\frac{i}{2} p^{\prime} q^{\prime}\right) \exp \left[\frac{s}{4}\left(q^{\prime 2}+p^{\prime 2}\right)\right],
$$

which is responsible for the mapping of bounded operators on the continuous phase-space, being $s$ a complex variable satisfying the condition $|s| \leq 1$. Here, the momentum and coordinate operators obey the Weyl-Heisenberg commutation relation $[\mathbf{Q}, \mathbf{P}]=i \mathbf{1}$. Since the above expression explicitly depends on $s$, this parameter labels an infinite family 
of mapping kernels. Each mapping kernel can be seen as the double Fourier transform of the displacement generators multiplied by a phase factor $\exp \left[(i / 2) p^{\prime} q^{\prime}\right]$ and by the folding function $\exp \left[(s / 4)\left(q^{\prime 2}+p^{2}\right)\right]$. For purposes which will become evident later, we write the mapping kernel as

$$
\mathbf{T}^{(s)}(q, p)=\int_{-\infty}^{\infty} \frac{d p^{\prime} d q^{\prime}}{2 \pi} \exp \left[-i p^{\prime}(q-\mathbf{Q})\right] \exp \left[i q^{\prime}(p-\mathbf{P})\right] \exp \left(-\frac{i}{2} p^{\prime} q^{\prime}\right)\left(\left\langle 0 \mid q^{\prime}, p^{\prime}\right\rangle\right)^{-s},
$$

where $\left|q^{\prime}, p^{\prime}\right\rangle$ is a coherent state.

The mapping of a given operator is achieved by the trace operation $\mathcal{O}^{(s)}(q, p)=\operatorname{Tr}\left[\mathbf{T}^{(s)}(q, p) \mathbf{O}\right]$, being $\mathcal{O}^{(s)}(q, p)$ the function which represents $\mathbf{O}$ in the associated usual phase-space. The mapping is one-to-one, and the operator is reobtained from its associated function by

$$
\mathbf{O}=\int_{-\infty}^{\infty} \frac{d p d q}{2 \pi} \mathcal{O}^{(s)}(q, p) \mathbf{T}^{(-s)}(q, p)
$$

It is clear that, for each operator, there is an infinite family of associated functions labeled by $s$. In particular, the phase-space representatives of the density operator are referred to as quasiprobability distributions functions and have, obviously, distinguishable importance [20]. One of the great virtues of the Cahill-Glauber approach is that three special and important types of quasidistributions, namely the Glauber-Sudarshan $(s=1)$, Wigner $(s=0)$ and Husimi functions $(s=-1)$, are particular cases. Each of these functions have been extensively explored and reviewed in the literature [23].

A particular mapping kernel, characterized by a given parameter $s$, can be expressed in terms of another mapping kernel with a different parameter value. The same holds true to the functions associated with a given operator. In fact, the procedure in the latter case can be easily shown to be the same as in the former. That is, we may discuss only the relation between the mapping kernels, knowing that equivalent relations are observed by the associated functions. In this way, the connection between the two mapping kernels is seen to be given by the trace of the product

$$
\begin{aligned}
\operatorname{Tr}\left[\mathbf{T}^{\left(s_{1}\right)}\left(q_{1}, p_{1}\right) \mathbf{T}^{\left(s_{2}\right)}\left(q_{2}, p_{2}\right)\right] & =\int_{-\infty}^{\infty} \frac{d q d p}{2 \pi} \exp \left\{i\left[q\left(p_{1}-p_{2}\right)-p\left(q_{1}-q_{2}\right)\right]\right\} \overbrace{\exp \left[\frac{s_{1}+s_{2}}{4}\left(q^{2}+p^{2}\right)\right]}^{(\langle 0 \mid q, p\rangle)-\left(s_{1}+s_{2}\right)} \\
& =\frac{-2}{s_{1}+s_{2}} \exp \left\{\frac{2}{s_{1}+s_{2}}\left[\left(p_{1}-p_{2}\right)^{2}+\left(q_{1}-q_{2}\right)^{2}\right]\right\} \quad \operatorname{Re}\left(s_{1}+s_{2}\right)<0 .
\end{aligned}
$$

We immediately recognize the important role played by the last exponential function in (3), since, if the condition $\operatorname{Re}\left(s_{1}+s_{2}\right)<0$ is not observed, the trace gives a divergent result. Thus, that condition imposes a constraint that defines a hierarchy. That is, on continuous phase space there is a hierarchical structure of mapping kernels allowing one to express a given phase-space function in terms of a Gaussian smoothing of another, and, as such, inverse relations do not exist. In other words, the Gaussian folding hierarchical structure observed by the quasidistributions has its roots in the functional form of $\langle 0 \mid q, p\rangle$. We stress this particular point as the discrete equivalent to equation (3) does not imply a hierarchical relation.

\section{THE DISCRETE MAPPING KERNEL}

\section{A. Preliminaries}

\section{Operator bases}

Long ago Schwinger proposed the following set of operators to act as a basis on an operator space

$$
\mathbf{S}(\eta, \xi)=\frac{1}{\sqrt{N}} \mathbf{U}^{\eta} \mathbf{V}^{\xi} \exp \left(\frac{\pi i}{N} \eta \xi\right)
$$

where the U's and V's are the so-called Schwinger unitary operators 24], $N$ is the dimension of the associated state space and the indices $\{\eta, \xi\}$ run on any complete set of residues $\bmod (N)$; in particular we choose the closed interval $[-\ell, \ell]$, with $\ell=(N-1) / 2$. For simplicity, we shall restrict ourselves to the odd $N$ case. Even dimensionalities, for the purposes of this paper, can also be dealt with simply by working on non-symmetrized intervals. 
The set $\{\mathbf{S}(\eta, \xi)\}_{\eta, \xi=-\ell, \ldots, \ell}$ spans a complete and orthonormal basis on the $N^{2}$ space of linear operators acting on finite complex vectorial spaces, in the sense that, as the trace operation stands as the inner product on operator spaces, any linear operator can be written as

$$
\mathbf{O}=\sum_{\eta, \xi=-\ell}^{\ell} \operatorname{Tr}\left[\mathbf{S}^{\dagger}(\eta, \xi) \mathbf{O}\right] \mathbf{S}(\eta, \xi)
$$

The fundamental result

$$
\operatorname{Tr}\left[\mathbf{S}^{\dagger}(\mu, \nu) \mathbf{S}(\eta, \xi)\right]=\delta_{\eta, \mu}^{[N]} \delta_{\xi, \nu}^{[N]}
$$

ensures that this decomposition is unique. The superscript $[N]$ on the Kroenecker deltas denotes that they are different from zero whenever their indices are $\bmod (N)$ congruent. The Schwinger basis elements also obey the property 6$]$

$$
\mathbf{S}^{\dagger}(\eta, \xi)=\mathbf{S}(-\eta,-\xi)
$$

\section{Discrete Coherent States}

The Schwinger operator bases elements also act as displacement operators on a particular reference state to form discrete coherent states as [4, 6]

$$
|\eta, \xi\rangle=\sqrt{N} \mathbf{S}(\eta,-\xi)|0,0\rangle
$$

where the reference state is written by means of the Jacobi $\vartheta_{3}$-function (whose explicit form is shown in Appendix A) as

$$
|0,0\rangle=\frac{1}{\mathcal{N}} \sum_{\gamma=-\ell}^{\ell} \vartheta_{3}(2 a \gamma \mid 2 i a)\left|u_{\gamma}\right\rangle
$$

where $\left\{\left|u_{\gamma}\right\rangle\right\}_{\gamma=-\ell, \ldots, \ell}$ are the eigenstates of the unitary operator $\mathbf{U}$,

$$
\mathcal{N}^{2}=\frac{1}{2 \sqrt{a}}\left[\vartheta_{3}(0 \mid i a) \vartheta_{3}(0 \mid 4 i a)+\vartheta_{4}(0 \mid i a) \vartheta_{2}(0 \mid 4 i a)\right]
$$

is the normalization constant, and $a=(2 N)^{-1}$. Due to the properties of the $\vartheta_{3}$-function, the reference state above is preserved under the action of the Fourier operator [6, 25]

$$
\mathfrak{F}|0,0\rangle=|0,0\rangle
$$

where

$$
\mathfrak{F}=\sum_{\gamma=-\ell}^{\ell}\left|v_{\gamma}\right\rangle\left\langle u_{\gamma}\right|
$$

and $\left\{\left|v_{\gamma}\right\rangle\right\}_{\gamma=-\ell, \ldots, \ell}$ are the eigenstates of $\mathbf{V}$, with $\left\langle u_{\mu} \mid v_{\gamma}\right\rangle=\exp [(2 \pi i / N) \mu \gamma]$. Parity of the $\vartheta_{3}$-function also ensures that $\left\langle u_{\kappa} \mid 0,0\right\rangle=\left\langle u_{-\kappa} \mid 0,0\right\rangle$, from which it follows

$$
\langle 0,0 \mid \mu, \nu\rangle=\langle 0,0 \mid-\mu,-\nu\rangle
$$

There are, of course, a number different recipes of discrete coherent states, some of them also in connection with $\vartheta$-functions, for instance [26, 27].

\section{B. The extended mapping kernel}

Now let us define the extended mapping kernel as

$$
\mathbf{S}^{(s)}(\eta, \xi)=\mathbf{S}(\eta, \xi)[\mathcal{K}(\eta, \xi)]^{-s}
$$


where $s$ is a complex number satisfying $|s| \leq 1$, and $\mathcal{K}(\eta, \xi)=\langle 0,0 \mid \eta, \xi\rangle$ denotes the overlap of coherent states explicitly calculated in Appendix A. The set $\{\mathbf{S}(\eta, \xi)\}_{\eta, \xi=-\ell, \ldots, \ell}$ itself spans a complete and orthogonal basis on operator space. Nevertheless, we can go back to decomposition (5), use equation (6), and introduce convenient factors to get

$$
\mathbf{O}=\sum_{\eta, \xi=-\ell}^{\ell} \operatorname{Tr}[\mathbf{S}(-\eta,-\xi) \mathbf{O}] \mathbf{S}(\eta, \xi) \underbrace{[\mathcal{K}(-\eta,-\xi)]^{s}[\mathcal{K}(\eta, \xi)]^{-s}}_{1},
$$

where equation (9) has been used. Conveniently grouping the terms the new decomposition reads

$$
\mathbf{O}=\sum_{\eta, \xi=-\ell}^{\ell} \operatorname{Tr}\left[\mathbf{S}^{(-s)}(-\eta,-\xi) \mathbf{O}\right] \mathbf{S}^{(s)}(\eta, \xi)
$$

Now, introducing the double Fourier transform of $\mathbf{S}^{(s)}(\eta, \xi)$, i.e.

$$
\mathbf{T}^{(s)}(\eta, \xi)=\frac{1}{\sqrt{N}} \sum_{\mu, \nu=-\ell}^{\ell} \mathbf{S}^{(s)}(\eta, \xi) \exp \left[-\frac{2 \pi i}{N}(\eta \mu+\xi \nu)\right]
$$

and its Fourier inverse, we can, after a few steps, write equation (10) as

$$
\mathbf{O}=\frac{1}{N} \sum_{\mu, \nu=-\ell}^{\ell} \mathcal{O}^{(-s)}(\mu, \nu) \mathbf{T}^{(s)}(\mu, \nu)
$$

with $\mathcal{O}^{(-s)}(\mu, \nu)=\operatorname{Tr}\left[\mathbf{T}^{(-s)}(\mu, \nu) \mathbf{O}\right]$, defining a one-to-one mapping between operators and functions defined on a discrete phase-space $\{\mu, \nu\}$, where explicitly

$$
\mathbf{T}^{(s)}(\mu, \nu)=\frac{1}{N} \sum_{\eta, \xi=-\ell}^{\ell} \mathbf{U}^{\eta} \mathbf{V}^{\xi} \exp \left[-\frac{2 \pi i}{N}(\eta \mu+\xi \nu)\right] \exp \left(\frac{\pi i}{N} \eta \xi\right)[\mathcal{K}(\eta, \xi)]^{-s}
$$

and $\mathcal{K}(\eta, \xi)$ can be shown to be a sum of products of Jacobi $\vartheta$-functions (as seen in Appendix A),

$$
\begin{aligned}
\mathcal{K}(\eta, \xi)= & \frac{1}{4 \sqrt{a} \mathcal{N}^{2}}\left\{\vartheta_{3}(a \eta \mid i a) \vartheta_{3}(a \xi \mid i a)+\vartheta_{3}(a \eta \mid i a) \vartheta_{4}(a \xi \mid i a) \exp (i \pi \eta)\right. \\
& \left.+\vartheta_{4}(a \eta \mid i a) \vartheta_{3}(a \xi \mid i a) \exp (i \pi \xi)+\vartheta_{4}(a \eta \mid i a) \vartheta_{4}(a \xi \mid i a) \exp [i \pi(\eta+\xi+N)]\right\} .
\end{aligned}
$$

The new kernel, written as in equation (12), allows us to conclude that the above sum of products of $\vartheta$-functions plays, in the discrete phase-space, the role reserved to the Gaussians in the continuous case.

\section{PROPERTIES}

\section{A. Basic general properties}

From the properties of the mapping kernel it is straightforward to obtain general properties of the associated functions in phase-space. We observe that all following properties correctly generalize the continuous CG ones. First we note that

$$
\text { (i) }\left[\mathbf{T}^{(s)}(\mu, \nu)\right]^{\dagger}=\mathbf{T}^{\left(s^{*}\right)}(\mu, \nu),
$$

implying that the mapping kernel is Hermitian for real values of the parameter $s$. As a direct consequence, the phase-space representatives of Hermitian operators are real.

Direct calculations also show that

$$
\begin{aligned}
& \text { (ii) } \frac{1}{N} \sum_{\mu, \nu=-\ell}^{\ell} \mathbf{T}^{(s)}(\mu, \nu)=\mathbf{1}, \\
& \text { (iii) } \operatorname{Tr}\left[\mathbf{T}^{(s)}(\mu, \nu)\right]=1, \\
& \text { (iv) } \operatorname{Tr}\left[\mathbf{T}^{(s)}(\mu, \nu) \mathbf{T}^{(-s)}\left(\mu^{\prime}, \nu^{\prime}\right)\right]=N \delta_{\mu, \mu^{\prime}}^{[N]} \delta_{\nu, \nu^{\prime}}^{[N]} .
\end{aligned}
$$


The property (iv) is a crucial one from which expression (11) could be immediately obtained. From this property also follows the general result

$$
\operatorname{Tr}(\mathbf{A B})=\frac{1}{N} \sum_{\mu, \nu=-\ell}^{\ell} \mathcal{A}^{(s)}(\mu, \nu) \mathcal{B}^{(-s)}(\mu, \nu)
$$

In fact, property (iv) is a particular case of the general expression

$$
\operatorname{Tr}\left[\mathbf{T}^{(s)}(\mu, \nu) \mathbf{T}^{(t)}\left(\mu^{\prime}, \nu^{\prime}\right)\right]=\frac{1}{N} \sum_{\eta, \xi=-\ell}^{\ell} \exp \left\{\frac{2 \pi i}{N}\left[\eta\left(\mu^{\prime}-\mu\right)+\xi\left(\nu^{\prime}-\nu\right)\right]\right\}[\mathcal{K}(\eta, \xi)]^{-(t+s)}
$$

which is the counterpart of equation (3). It must be stressed that this expression is always well defined, even for $\operatorname{Re}(t+s)<0$, as $\mathcal{K}(\eta, \xi) \neq 0$.

\section{B. Particular cases}

There are three important particular cases to be discussed:

i) $(\mathrm{s}=0)$ In such a case it is easy to see that

$$
\mathbf{T}^{(0)}(\mu, \nu)=\mathbf{G}(\mu, \nu)
$$

where $\mathbf{G}(\mu, \nu)$ is the mapping kernel introduced by Galetti and Piza, which is a discrete generalization of the Weyl-Wigner mapping kernel [2, 4, 28, 29]. In that case, being $\boldsymbol{\rho}$ the density operator, equation (11) would read, for $\mathbf{O}=\boldsymbol{\rho}$,

$$
\boldsymbol{\rho}=\frac{1}{N} \sum_{\mu, \nu=-\ell}^{\ell} \mathcal{W}(\mu, \nu) \mathbf{G}(\mu, \nu)
$$

with $\mathcal{W}(\mu, \nu)=\operatorname{Tr}[\mathbf{G}(\mu, \nu) \boldsymbol{\rho}]$ a discrete Wigner function.

ii) $(\mathrm{s}=-1)$ A fundamental property of our mapping kernel is

$$
\mathbf{T}^{(-1)}(\mu, \nu)=|\mu, \nu\rangle\langle\mu, \nu|
$$

which can be proved decomposing the coherent state projector in the Schwinger operator basis as (using equations (5) and (6)

$$
|\mu, \nu\rangle\langle\mu, \nu|=\frac{1}{N} \sum_{\eta, \xi=-\ell}^{\ell} \mathbf{U}^{\eta} \mathbf{V}^{\xi} \exp \left(\frac{i \pi}{N} \eta \xi\right) \operatorname{Tr}\left[|\mu, \nu\rangle\langle\mu, \nu| \mathbf{V}^{-\xi} \mathbf{U}^{-\eta} \exp \left(-\frac{i \pi}{N} \eta \xi\right)\right]
$$

which, by applying the definition of the coherent states, equation (7), reads

$$
|\mu, \nu\rangle\langle\mu, \nu|=\frac{1}{N} \sum_{\eta, \xi=-\ell}^{\ell} \mathbf{U}^{\eta} \mathbf{V}^{\xi}\left\langle 0,0\left|\mathbf{V}^{\nu} \mathbf{U}^{-\mu} \mathbf{V}^{-\xi} \mathbf{U}^{-\eta} \mathbf{U}^{\mu} \mathbf{V}^{-\nu}\right| 0,0\right\rangle
$$

and using the Weyl commutation relation, $\mathbf{U}^{\alpha} \mathbf{V}^{\beta}=\exp [-(2 \pi i / N) \alpha \beta] \mathbf{V}^{\beta} \mathbf{U}^{\alpha}$,

$$
|\mu, \nu\rangle\langle\mu, \nu|=\frac{1}{N} \sum_{\eta, \xi=-\ell}^{\ell} \mathbf{U}^{\eta} \mathbf{V}^{\xi} \exp \left[-\frac{2 \pi i}{N}(\eta \mu+\xi \nu)\right] \exp \left(\frac{\pi i}{N} \eta \xi\right)\langle 0,0 \mid \eta, \xi\rangle,
$$

where in the last step parity of $\langle 0,0 \mid \eta, \xi\rangle$ with respect to $\eta$ was used. This proves our assertion. As a consequence, the phase-space decomposition of the density operator, associated with this particular value of the parameter $s$, reads

$$
\boldsymbol{\rho}=\frac{1}{N} \sum_{\mu, \nu=-\ell}^{\ell} P(\mu, \nu)|\mu, \nu\rangle\langle\mu, \nu|
$$

allowing us to identify $P(\mu, \nu)$ as a discrete Glauber-Sudarshan distribution. 
iii) $\quad(\mathrm{s}=1)$ In this case we may write

$$
\boldsymbol{\rho}=\frac{1}{N} \sum_{\mu, \nu=-\ell}^{\ell} \operatorname{Tr}\left[\mathbf{T}^{(-1)}(\mu, \nu) \boldsymbol{\rho}\right] \mathbf{T}^{(1)}(\mu, \nu)
$$

which is simply

$$
\boldsymbol{\rho}=\frac{1}{N} \sum_{\mu, \nu=-\ell}^{\ell} \mathcal{H}(\mu, \nu) \mathbf{T}^{(1)}(\mu, \nu)
$$

By definition $\mathcal{H}(\mu, \nu)=\langle\mu, \nu|\boldsymbol{\rho}| \mu, \nu\rangle$ is positive definite, and it can be identified as a discrete Husimi function.

As any operator can be decomposed by the use of expression (11), it follows that we are allowed to write

$$
\mathbf{T}^{(-1)}(\mu, \nu)=\frac{1}{N} \sum_{\sigma, \lambda=-\ell}^{\ell} \operatorname{Tr}\left[\mathbf{T}^{(-0)}(\sigma, \lambda) \mathbf{T}^{(-1)}(\mu, \nu)\right] \mathbf{T}^{(0)}(\sigma, \lambda),
$$

where the minus signal was kept only for clarity. We then use equation (18) to write explicitly

$$
\operatorname{Tr}\left[\mathbf{T}^{(0)}(\sigma, \lambda) \mathbf{T}^{(-1)}(\mu, \nu)\right]=\frac{1}{N} \sum_{\eta, \xi=-\ell}^{\ell} \exp \left\{\frac{2 \pi i}{N}[\eta(\mu-\sigma)+\xi(\nu-\lambda)]\right] \mathcal{K}(\eta, \xi),
$$

that is, the discrete Fourier transform of the $\mathcal{K}(\eta, \xi)$ is the folding function. The above result can also be written in the compact form

$$
\operatorname{Tr}\left[\mathbf{T}^{(0)}(\sigma, \lambda) \mathbf{T}^{(-1)}(\mu, \nu)\right]=\langle\mu, \nu|\mathbf{G}(\sigma, \lambda)| \mu, \nu\rangle,
$$

which is precisely the Wigner function associated with a coherent state $|\mu, \nu\rangle$. We therefore have

$$
\mathbf{T}^{(-1)}(\mu, \nu)=\frac{1}{N} \sum_{\sigma, \lambda=-\ell}^{\ell}\langle\mu, \nu|\mathbf{G}(\sigma, \lambda)| \mu, \nu\rangle \mathbf{T}^{(0)}(\sigma, \lambda) .
$$

In the same form we now decompose $\mathbf{T}^{(0)}(\mu, \nu)$ as

$$
\mathbf{T}^{(0)}(\mu, \nu)=\frac{1}{N} \sum_{\sigma, \lambda=-\ell}^{\ell} \operatorname{Tr}\left[\mathbf{T}^{(0)}(\sigma, \lambda) \mathbf{T}^{(-1)}(\mu, \nu)\right] \mathbf{T}^{(1)}(\sigma, \lambda),
$$

which allows us to use once again the above result for the trace and write

$$
\mathbf{T}^{(0)}(\mu, \nu)=\frac{1}{N} \sum_{\sigma, \lambda=-\ell}^{\ell}\langle\mu, \nu|\mathbf{G}(\sigma, \lambda)| \mu, \nu\rangle \mathbf{T}^{(1)}(\sigma, \lambda) .
$$

Multiplying both equations (22) and (23) by the density operator $\rho$ and taking the trace, we are led to the suggestive results

$$
\begin{aligned}
& \mathcal{H}(\mu, \nu)=\frac{1}{N} \sum_{\sigma, \lambda=-\ell}^{\ell}\langle\mu, \nu|\mathbf{G}(\sigma, \lambda)| \mu, \nu\rangle \mathcal{W}(\sigma, \lambda), \\
& \mathcal{W}(\mu, \nu)=\frac{1}{N} \sum_{\sigma, \lambda=-\ell}^{\ell}\langle\mu, \nu|\mathbf{G}(\sigma, \lambda)| \mu, \nu\rangle P(\sigma, \lambda),
\end{aligned}
$$

which are the discrete counterparts of the well known Gaussian smoothing that occurs in the continuous case, in agreement with the hierarchy present in that context. 
It must be stressed, however, that, opposed to the continuous case, it is now possible to write

$$
\begin{aligned}
& \mathbf{T}^{(0)}(\mu, \nu)=\frac{1}{N} \sum_{\sigma, \lambda=-\ell}^{\ell} \Lambda(\mu-\sigma, \nu-\lambda) \mathbf{T}^{(-1)}(\sigma, \lambda) \\
& \mathbf{T}^{(1)}(\mu, \nu)=\frac{1}{N} \sum_{\sigma, \lambda=-\ell}^{\ell} \Lambda(\mu-\sigma, \nu-\lambda) \mathbf{T}^{(0)}(\sigma, \lambda),
\end{aligned}
$$

where

$$
\Lambda(\mu-\sigma, \nu-\lambda)=\frac{1}{N} \sum_{\eta, \xi=-\ell}^{\ell} \exp \left\{\frac{2 \pi i}{N}[\eta(\mu-\sigma)+\xi(\nu-\lambda)]\right\}[\mathcal{K}(\eta, \xi)]^{-1},
$$

which, at least in principle, can always be calculated (we remind again that $\mathcal{K}(\eta, \xi)$ is finite and different from zero).

Also very illustrative is the result that follows from the decomposition

$$
\mathbf{T}^{(-1)}(\mu, \nu)=\frac{1}{N} \sum_{\sigma, \lambda=-\ell}^{\ell} \operatorname{Tr}\left[\mathbf{T}^{(-1)}(\sigma, \lambda) \mathbf{T}^{(-1)}(\mu, \nu)\right] \mathbf{T}^{(1)}(\sigma, \lambda) .
$$

With

$$
\operatorname{Tr}\left[\mathbf{T}^{(-1)}(\sigma, \lambda) \mathbf{T}^{(-1)}(\mu, \nu)\right]=\frac{1}{N} \sum_{\eta, \xi=-\ell}^{\ell} \exp \left\{\frac{2 \pi i}{N}[\eta(\mu-\sigma)+\xi(\nu-\lambda)]\right\}[\mathcal{K}(\eta, \xi)]^{2},
$$

which can be shown to be $|\langle\mu, \nu \mid \sigma, \lambda\rangle|^{2}$, we have

$$
\mathbf{T}^{(-1)}(\mu, \nu)=\frac{1}{N} \sum_{\sigma, \lambda=-\ell}^{\ell}|\langle\mu, \nu \mid \sigma, \lambda\rangle|^{2} \mathbf{T}^{(1)}(\sigma, \lambda) .
$$

Thus $|\langle\mu, \nu \mid \sigma, \lambda\rangle|^{2}$ itself, which is the Husimi function associated with the discrete coherent-state $|\mu, \nu\rangle$, acts here as the smoothing function.

\section{CONTINUUM LIMIT}

Following the procedure detailed in both [28, 29], the continuum limit of the mapping kernel (12) is reached as follows: we introduce the scaling parameter $\epsilon=(2 \pi / N)^{1 / 2}$, which will become infinitesimal as $N \rightarrow \infty$, and the two Hermitian operators

$$
\mathbf{P}=\sum_{\mu=-\ell}^{\ell} \mu \epsilon p_{0}\left|v_{\mu}\right\rangle\left\langle v_{\mu}\left|\quad \mathbf{Q}=\sum_{\mu^{\prime}=-\ell}^{\ell} \mu^{\prime} \epsilon q_{0}\right| u_{\mu^{\prime}}\right\rangle\left\langle u_{\mu^{\prime}}\right|,
$$

constructed out of the projectors of the eigenstates of $\mathbf{U}$ and $\mathbf{V}$. The parameters $p_{0}$ and $q_{0}$, with $p_{0} q_{0}=\hbar=1$, are chosen to be real, carrying units of momentum and position, respectively, while $\epsilon p_{0}$ and $\epsilon q_{0}$ are the distance between successive eigenvalues of the $\mathbf{P}$ and $\mathbf{Q}$ operators. Then, rewriting the Schwinger operators as

$$
\mathbf{V}=\exp \left(\frac{i \epsilon \mathbf{P}}{p_{0}}\right) \quad \mathbf{U}=\exp \left(\frac{i \epsilon \mathbf{Q}}{q_{0}}\right)
$$

and performing the change of variables $q^{\prime}=-q_{0} \epsilon \xi, p^{\prime}=p_{0} \epsilon \eta, p=p_{0} \epsilon \nu$ and $q=q_{0} \epsilon \mu$, we obtain

$$
\mathbf{T}^{(s)}(q, p)=\sum_{q^{\prime}=-q_{0} \epsilon \ell}^{q_{0} \epsilon \ell} \sum_{p^{\prime}=-p_{0} \epsilon \ell}^{p_{0} \epsilon \ell} \frac{\Delta q^{\prime} \Delta p^{\prime}}{2 \pi} \exp \left[-i p^{\prime}(q-\mathbf{Q})\right] \exp \left[i q^{\prime}(p-\mathbf{P})\right]\left[\mathcal{K}\left(p^{\prime} / p_{0} \epsilon,-q^{\prime} / q_{0} \epsilon\right)\right]^{-s} \exp \left(-\frac{i}{2} q^{\prime} p^{\prime}\right) .
$$

As $N \rightarrow \infty$, it follows that $\Delta q^{\prime} \rightarrow d q^{\prime}$ and $\Delta p^{\prime} \rightarrow d p^{\prime}$. Since the continuum limit of the discrete coherent-states has been already discussed in [4, 6$]$, it is clear that the term $\left[\mathcal{K}\left(p^{\prime} / p_{0} \epsilon,-q^{\prime} / q_{0} \epsilon\right)\right]^{-s}$, which is even, will go to $\left(\left\langle 0 \mid q^{\prime}, p^{\prime}\right\rangle\right)^{-s}$. Therefore we end up with

$$
\mathbf{T}^{(s)}(q, p)=\int_{-\infty}^{\infty} \frac{d p^{\prime} d q^{\prime}}{2 \pi} \exp \left[-i p^{\prime}(q-\mathbf{Q})\right] \exp \left[i q^{\prime}(p-\mathbf{P})\right] \exp \left(-\frac{i}{2} p^{\prime} q^{\prime}\right)\left(\left\langle 0 \mid q^{\prime}, p^{\prime}\right\rangle\right)^{-s},
$$

which is exactly the mapping kernel (2) of Cahill and Glauber. 


\section{CONCLUDING REMARKS}

The results obtained here show a genuine discrete mathematical structure which closely parallels the one of Cahill and Glauber. This was achieved pursuing the lines proposed in [2], which makes use of the discrete Fourier transform of the Schwinger operator basis, to deal with the discrete phase-space problem. Now, we stress that expression (2) is as simple as it is important, since it clarifies the role of the coherent states overlap within the CG approach. By its turn, the discrete coherent states proposed in [4, 6, 26, 27] provide a natural path for a discrete extension of the CG formalism, while the properties of these states have played a crucial role as they led, for example to the basic equation (20). Thus, the coherent states overlap can be seen as the link between the discrete and continuous approaches. Furthermore, the continuum limit presented in section V ensures that the CG mapping scheme is correctly recovered through a limiting procedure which is mathematically consistent 29, 30, 31, 32].

It is worth mentioning that Opatrný et al [5] have pursued a goal similar to ours. Although both approaches share virtues, our formalism presents mathematical features that allow us to achieve farther reaching results. It is precisely the correct choice for the reference state, and the mathematical procedure adopted here, that lead to the obtention of such a wide set of important results.

The use of Schwinger operators is crucial if one is concerned with the problem of ordering. As they are unitary shift operators, equation (12) makes it clear that the associated expansion is necessarily linked to a particular ordering of $\mathbf{U}$ and $\mathbf{V}$, which can be directly connected to the $\mathbf{Q}$ and $\mathbf{P}$ ordering of the continuous case.

Concerning the role of the Jacobi Theta functions in the discrete phase-space context - they are implicit in the $\mathcal{K}(\eta, \xi)$ term -, comparison with the usual CG results makes it evident that the Gaussian (or anti-Gaussian) terms, which are present in the continuous case, are here replaced by the sum of products of $\vartheta$-functions (13), and its Fourier transform (21); both play here the role of the smoothing functions.

It is always important to emphasize the discrete case's peculiar features that do not have correspondence in the continuum. A plain example of these is expressed by the well-behaved function given by equation (24), whose continuum limit clearly diverges, as the hierarchical structure presented in (4) would imply. The finite character of the discrete scenario prevents such a behaviour since, even if some terms in equation (24) might become large for large $N$, they remain always finite due to the behaviour of the $\vartheta$-functions. This allows one - to give a extreme example to express, in the discrete scenario, the Glauber-Sudarshan function in terms of the Husimi function.

Finally, it is worth mentioning that the mathematical formalism developed here opens new possibilities of investigations in quantum tomography and quantum teleportation. These considerations are under current research and will be published elsewhere (for instance, see reference [22]).

\section{Acknowledgments}

This work has been supported by Fundação de Amparo à Pesquisa do Estado de São Paulo (FAPESP), Brazil, project nos. 03/13488-0 (MR), 01/11209-0 (MAM), and 00/15084-5 (MAM and MR). DG acknowledges partial financial support from the Conselho Nacional de Desenvolvimento Científico e Tecnológico (CNPq), Brazil.

\section{APPENDIX A: THE DISCRETE COHERENT-STATE OVERLAP}

The vacuum associated with the discrete coherent-states (7), given by equation (8), is written in terms of the Jacobi $\vartheta_{3}$-function, which reads

$$
\vartheta_{3}(a \mu \mid i a)=\sum_{\alpha=-\infty}^{\infty} \exp \left(-a \pi \alpha^{2}+2 \pi i a \mu \alpha\right)
$$

with $a=(2 N)^{-1}$. Explicitly, then, the overlap $\langle\eta, \xi \mid \mu, \nu\rangle$ is:

$$
\begin{aligned}
\langle\eta, \xi \mid \mu, \nu\rangle & =\frac{1}{\mathcal{N}^{2}} \exp [2 \pi i a(-\mu \nu+\eta \xi)]\left\langle 0,0\left|\mathbf{V}^{\xi} \mathbf{U}^{-\eta} \mathbf{U}^{\mu} \mathbf{V}^{-\nu}\right| 0,0\right\rangle \\
& =\frac{1}{\mathcal{N}^{2}} \exp \{2 \pi i a[-\mu \nu+\eta \xi+2 \xi(\mu-\eta)]\} \sum_{\kappa, \nu=-\ell}^{\ell} \vartheta_{3}(2 a \kappa \mid 2 i a) \vartheta_{3}(2 a \nu \mid 2 i a)\left\langle v_{\nu}\left|\mathbf{U}^{\mu-\eta} \mathbf{V}^{\xi-\nu}\right| v_{\kappa}\right\rangle,
\end{aligned}
$$


which, by evaluating the matrix element within the summation, gives

$$
\frac{1}{\mathcal{N}^{2}} \exp \{2 \pi i a[-\mu \nu+\eta \xi+2 \xi(\mu-\eta)]\} \sum_{\kappa=-\ell}^{\ell} \vartheta_{3}(2 a \kappa \mid 2 i a) \vartheta_{3}(2 a(\kappa+\mu-\eta) \mid 2 i a) \exp [-\pi i a \kappa(\nu-\xi)] .
$$

Now let us call

$$
A(\mu, \nu)=\sum_{\kappa=-\ell}^{\ell} \vartheta_{3}(2 a \kappa \mid 2 i a) \vartheta_{3}(2 a(\kappa+\mu) \mid 2 i a) \exp (-\pi i a \kappa \nu)
$$

so we write

$$
\langle\eta, \xi \mid \mu, \nu\rangle=\frac{1}{\mathcal{N}^{2}} \exp \{2 \pi i a[-\mu \nu+\eta \xi+2 \xi(\mu-\eta)]\} A(\mu-\eta, \nu-\xi) .
$$

The task now is to evaluate explicitly the term $A(\mu, \nu)$. Prior to that, some properties of $A(\mu, \nu)$ might be verified from the start. First, we note that $A(\mu, \nu)=A(\nu, \mu)$ (using the fact that the $\vartheta_{3}$ is an eigenfunction of the discrete Fourier transform), and also $A\left(\mu+N \kappa, \nu+N \kappa^{\prime}\right)=A(\mu, \nu)$, for $\kappa$ and $\kappa^{\prime}$ integers.

Let us now evaluate $A(\mu, \nu)$. We start using the $\vartheta_{3}$ definition to get

$$
A(\mu, \nu)=\sum_{\kappa=-\ell}^{\ell} \sum_{\alpha, \beta=-\infty}^{\infty} \exp (-4 \pi i a \kappa \mu) \exp \left(-2 \pi a \alpha^{2}+4 \pi i a \kappa \alpha\right) \exp \left[-2 \pi a \beta^{2}+4 \pi i a(\kappa+\nu) \beta\right] .
$$

The sum over $\kappa$ can be readily carried out, which gives us $N \delta_{\beta, \mu-\alpha}^{[N]}$. Thus, $\beta$ will assume the values $\mu-\alpha+N \gamma$, where $\gamma$ is an arbitrary integer, yielding

$$
N \sum_{\alpha, \gamma=-\infty}^{\infty} \exp \left(-2 \pi a \alpha^{2}\right) \exp \left[-2 \pi a(\mu-\alpha+N \gamma)^{2}+4 \pi i a \nu(\mu-\alpha+N \gamma)\right] .
$$

The last term in the second exponential is equal to one, and we shift the sum over $\alpha$ by $\mu$ in order to get

$$
N \sum_{\alpha, \gamma=-\infty}^{\infty} \exp \left[-4 \pi a\left(\alpha-\frac{\gamma N}{2}\right)^{2}-\frac{\pi \gamma^{2} N}{2}-2 \pi a \mu^{2}-4 \pi i a \alpha(\nu-i \mu)\right]
$$

Now, we split the sum over $\gamma$ in contributions coming from the even (e) and odd (o) integers as follows: $A=A_{e}+A_{o}$. Consequently, the even term can be dealt with by shifting the sum over $\alpha$ by $-N \gamma$,

$$
\begin{aligned}
A_{e}(\mu, \nu) & =N \sum_{\alpha, \gamma=-\infty}^{\infty} \exp \left[-4 \pi a \alpha^{2}-2 \pi \gamma^{2} N-2 \pi a \mu^{2}+4 \pi i a(\alpha+N \gamma)(\nu-i \mu)\right] \\
& =N \sum_{\alpha=-\infty}^{\infty} \exp \left[-4 \pi a \alpha^{2}+4 \pi i a \alpha(\nu-i \mu)\right] \sum_{\gamma=-\infty}^{\infty} \exp \left[-2 \pi \gamma^{2} N+4 \pi i a \gamma N(\nu-i \mu)\right] \\
& =N \vartheta_{3}(2 a(\nu-i \mu) \mid 4 i a) \vartheta_{3}(i \mu \mid 2 i N) \exp \left(-2 \pi a \mu^{2}\right),
\end{aligned}
$$

and, in a similar fashion, the odd term gives

$$
A_{o}(\mu, \nu)=N \vartheta_{3}(2 a[\nu-i(\mu+N)] \mid 4 i a) \vartheta_{2}(i \mu \mid 2 i N) \exp \left(-\frac{\pi N}{2}-\pi \mu-2 \pi a \mu^{2}\right) .
$$

Next, using a relation (all the following relations for $\vartheta$-functions come from $\underline{33}$ ])

$$
\vartheta_{3}\left(\varsigma+\frac{\tau}{2} \mid \tau\right)=\exp \left(-i \pi \frac{\tau}{4}-i \pi \varsigma\right) \vartheta_{2}(\varsigma \mid \tau)
$$

we get

$$
A_{o}(\mu, \nu)=N \vartheta_{3}(2 a[\nu-i(\mu+N)] \mid 4 i a) \vartheta_{3}(i(\mu+N) \mid 2 i N) \exp \left[-2 \pi a(\mu+N)^{2}\right] .
$$


Then

$$
\begin{aligned}
A(\mu, \nu)= & N \vartheta_{3}(2 a[\nu-i \mu] \mid 4 i a) \vartheta_{3}(i \mu \mid 2 i N) \exp \left(-2 a \pi \mu^{2}\right) \\
& +N \vartheta_{3}(2 a[\nu-i(\mu+N)] \mid 4 i a) \vartheta_{3}(i(\mu+N) \mid 2 i N) \exp \left[-2 \pi a(\mu+N)^{2}\right] .
\end{aligned}
$$

Now we recall the fundamental property of $\vartheta$-functions,

$$
\vartheta_{3}\left(\frac{\varsigma}{i \tau} \mid \frac{i}{\tau}\right)=\sqrt{\tau} \exp \left(\frac{\pi \varsigma^{2}}{\tau}\right) \vartheta_{3}(\varsigma \mid i \tau)
$$

which applied to the $\vartheta_{3}(i \mu \mid 2 i N)$ and $\vartheta_{3}(i(\mu+N) \mid 2 i N)$ terms above gives

$$
\begin{aligned}
A(\mu, \nu)= & \sqrt{\frac{N}{2}} \vartheta_{3}(2 a(\nu-i \mu) \mid 4 i a) \vartheta_{3}(a \mu \mid i a) \exp \left(-\pi a \mu^{2}\right) \\
& +\sqrt{\frac{N}{2}} \vartheta_{3}(2 a[\nu-i(\mu+N)] \mid 4 i a) \vartheta_{3}(a(\mu+N) \mid i a) \exp \left[-\pi a(\mu+N)^{2}\right] .
\end{aligned}
$$

Still, one has to use the relation

$$
\vartheta_{3}(\varsigma \mid \tau)=\frac{1}{2}\left[\vartheta_{3}\left(\frac{\varsigma}{2} \mid \frac{\tau}{4}\right)+\vartheta_{4}\left(\frac{\varsigma}{2} \mid \frac{\tau}{4}\right)\right]
$$

in the first $\vartheta$-function of each of the two terms above to finally employ the relations of quasi-periodicity

$$
\begin{aligned}
& \vartheta_{3}(\varsigma+m \tau \mid \tau)=\exp \left(-\pi i \tau m^{2}+2 \pi i m \varsigma\right) \vartheta_{3}(\varsigma \mid \tau) \\
& \vartheta_{4}(\varsigma+m \tau \mid \tau)=\exp \left(-\pi i \tau m^{2}+2 \pi i m \varsigma+\pi i m\right) \vartheta_{4}(\varsigma \mid \tau),
\end{aligned}
$$

to reach the desired result

$$
\begin{aligned}
A(\mu, \nu)= & \frac{1}{4 \sqrt{a}} \exp (2 \pi i a \mu \nu)\left\{\vartheta_{3}(a \mu \mid i a) \vartheta_{3}(a \nu \mid i a)+\vartheta_{3}(a \mu \mid i a) \vartheta_{4}(a \nu \mid i a) \exp (i \pi \mu)\right. \\
& \left.+\vartheta_{4}(a \mu \mid i a) \vartheta_{3}(a \nu \mid i a) \exp (i \pi \nu)+\vartheta_{4}(a \mu \mid i a) \vartheta_{4}(a \nu \mid i a) \exp [i \pi(\nu+\mu+N)]\right\} .
\end{aligned}
$$

This completes the calculation of $\langle\eta, \xi \mid \mu, \nu\rangle$.

Finally, it is convenient to write down the result for the particular case $\mathcal{K}(\mu, \nu) \equiv\langle 0,0 \mid \mu, \nu\rangle$, i.e.,

$$
\mathcal{K}(\mu, \nu)=\frac{1}{\mathcal{N}^{2}} \exp (-2 \pi i a \mu \nu) A(\mu, \nu)
$$

[1] Wootters W K 1987 A Wigner-function formulation of finite-state quantum mechanics Ann. Phys. (NY) 1761

[2] Galetti D and de Toledo Piza A F R 1988 An extended Weyl-Wigner transformation for special finite spaces Physica A 149267

[3] Cohendet O, Combe P, Sirugue M and Sirugue-Collin M 1988 A stochastic treatment of the dynamics of an integer spin J. Phys. A: Math. Gen. 212875

[4] Galetti D and de Toledo Piza A F R 1992 Discrete quantum phase spaces and the $\bmod (N)$ invariance Physica A 186513

[5] Opatrný T, Welsch D-G and Bužek V 1996 Parametrized discrete phase-space functions Phys. Rev. A 533822

[6] Galetti D and Marchiolli M A 1996 Discrete coherent states and probability distributions in finite-dimensional spaces Ann. Phys. (NY) 249454 Marchiolli M A 2003 Nonclassical statistical properties of finite-coherent states in the framework of the Jaynes-Cummings model Physica A 319331

[7] Luis A and Peřina J 1998 Discrete Wigner function for finite-dimensional systems J. Phys. A: Math. Gen. 31 1423

[8] Hakioğlu T 1998 Finite-dimensional Schwinger basis, deformed symmetries, Wigner function, and an algebraic approach to quantum phase J. Phys. A: Math. Gen. 316975

[9] Mukunda N, Chatuverdi S and Simon R 2004 Wigner distributions for non-Abelian finite groups of odd order Phys. Lett. A 321160

[10] Gibbons K S, Hoffman M J and Wootters W K 2004 Discrete phase space based on finite fields Phys. Rev. A 70 062101

[11] Vourdas A 2004 Quantum systems with finite Hilbert space Rep. Prog. Phys. 67267 and references therein 
[12] Leonhardt U 1995 Quantum-state tomography and discrete Wigner functions Phys. Rev. Lett. 744101 Leonhardt U 1996 Discrete Wigner function and quantum-state tomography Phys. Rev. A 532998

[13] Miquel C, Paz J P, Saraceno M, Knill E, Laflamme R and Negrevergne C 2002 Interpretation of tomography and spectroscopy and dual forms of quantum computation Nature (London) $\mathbf{4 1 8} 59$

Paz J P and Roncaglia A J 2003 Quantum gate arrays can be programmed to evaluate the expectation value of any operator Phys. Rev. A 68052316

Paz J P, Roncaglia A J and Saraceno M 2004 Quantum algorithms for phase-space tomography Phys. Rev. A 69032312

[14] Koniorczyk M, Bužek V and Janszky J 2001 Wigner-function description of quantum teleportation in arbitrary dimensions and a continuous limit Phys. Rev. A 64034301

[15] Paz J P 2002 Discrete Wigner functions and the phase-space representation of quantum teleportation Phys. Rev. A 65 062311

[16] Miquel C, Paz J P and Saraceno M 2002 Quantum computers in phase space Phys. Rev. A 65062309

Bianucci P, Miquel C, Paz J P and Saraceno M 2002 Discrete Wigner functions and the phase space representation of quantum computers Phys. Lett. A 297353

[17] Bianucci P, Paz J P and Saraceno M 2002 Decoherence for classically chaotic quantum maps Phys. Rev. E 65046226

López C C and Paz J P 2003 Phase-space approach to the study of decoherence in quantum walks Phys. Rev. A 68052305 Aolita M L, García-Mata I and Saraceno M 2004 Noise models for superoperators in the chord representation Phys. Rev. A $\mathbf{7 0} 062301$

[18] Paz J P, Roncaglia A J and Saraceno M 2004 Qubits in phase space: Wigner function approach to quantum error correction and the mean king problem Preprint quant-ph/0410117

[19] Galvão E F 2005 Discrete Wigner functions and quantum computational speedup Phys. Rev. A 71042302

[20] Hillery M, O'Connell R F, Scully M O and Wigner E P 1984 Distribution functions in Physics: Fundamentals Phys. Rep. 106121

Lee H-W 1995 Theory and application of the quantum phase-space distribution functions Phys. Rep. 259147

[21] Cahill K E and Glauber R J 1969 Ordered expansions in boson amplitude operators Phys. Rev. 1771857

Cahill K E and Glauber R J 1969 Density operators and quasiprobability distributions Phys. Rev. 1771882

[22] Marchiolli M A, Ruzzi M and Galetti D 2005 Extended Cahill-Glauber formalism for finite-dimensional spaces: II. Applications in quantum tomography and quantum teleportation Preprint quant-ph/0504107

[23] See, for instance, Schleich W P 2001 Quantum optics in Phase-Space (Berlin: Wiley-VCH)

[24] Schwinger J 2000 Advanced Book Classics: Quantum Kinematics and Dynamics (New York: Perseus Publishing)

Schwinger J 2001 Quantum Mechanics: Symbolism of Atomic Measurements (New York: Springer-Verlag)

[25] Mehta M L 1987 Eigenvalues and eigenvectors of the finite Fourier transform J. Math. Phys. 28781

[26] Zhang S and Vourdas A 2004 Analytic representation of finite quantum systems J. Phys. A: Math. Gen. 378349

[27] Leboeuf P and Voros A 1990 Chaos-revealing multiplicative representation of quantum eigenstates J. Phys. A: Math. Gen. 231765

[28] Ruzzi M and Galetti D 2000 Quantum discrete phase space dynamics and its continuous limit J. Phys. A: Math. Gen. 33 1065

[29] Ruzzi M and Galetti D 2002 Schwinger and Pegg-Barnett approaches and a relationship between angular and Cartesian quantum descriptions: II. Phase spaces J. Phys. A: Math. Gen. 354633

[30] Barker L 2001 Continuum quantum systems as limits of discrete quantum systems: I. State Vectors J. Funct. Anal. 186 153

[31] Barker L 2001 Continuum quantum systems as limits of discrete quantum systems: II. State functions J. Phys A: Math. Gen. 344673

[32] Barker L 2001 Continuum quantum systems as limits of discrete quantum systems: III. Operators J. Math. Phys. 42 4653

[33] Vilenkin N J and Klimyk A U 1992 Representation of Lie Groups and Special Functions: Simplest Lie Groups, Special Functions and Integral Transforms (Dordrecht: Kluwer Academic Publications)

Bellman R 1961 A Brief Introduction to Theta Functions (New York: Holt, Rinehart and Winston)

Magnus W, Oberhettinger F and Soni R P 1966 Formulas and Theorems for the Special Functions of Mathematical Physics (New York: Springer-Verlag)

Whittaker E T and Watson G N 2000 Cambridge Mathematical Library: A course of modern analysis (United Kingdom: Cambridge University Press) 